Pavle Sekeruš ${ }^{*}$

Ivana Živančević Sekeruš

Faculté de Philosophie et Lettres,

Université de Novi Sad
UDK : 52 :821.163.41.09 Milanković M.

821.133.1.09 Fontenelle B.

DOI: $10.19090 /$ gff.2021.3.193-202

originalni naučni rad

\title{
LA LECTURE FRANÇAISE DE MILUTIN MILANKOVIĆ
}

Il y aurait eu peu de chance que les œuvres de Milutin Milanković, mathématicien, astronome et climatologue qui, pour les non-initiés, portent des titres énigmatiques et parlent des phénomènes thermiques et de la radiation solaire attirent l'attention des littéraires, si parmi elles on n'avait pas trouvé quelques œuvres de vulgarisation scientifique, destinées au grand public. L'une d'elles, intitulée Kroz vasionu i vekove (À travers l'univers et les siècles) de 1928, roman épistolaire de vulgarisation, dévoile assez rapidement la lecture française de l'auteur. L'influence de Bernard Le Bovier de Fontenelle et de son livre Entretiens sur la pluralité des mondes de 1686 paraît évidente. Ni les historiens des sciences, ni les historiens de la littérature n'en disent pas grand-chose. L'analyse comparative de ses deux ouvrages qui, utilisant des procédés semblables, explorent des zones frontalières entre la science et la fiction du XVII et du $\mathrm{XX}^{\mathrm{e}}$ siècle, fait l'objet de ce travail. Nos objectifs dans cet article étaient de replacer les ouvrages présentés dans le contexte scientifique et culturel de l'époque, puis de jeter un regard nouveau sur des textes littéraires à forte teneur scientifique, analyser le phénomène de vulgarisation scientifique et enfin de mettre en évidence les rapports souvent méconnus entre littérature et science.

Mots clés : Milanković, Fontenelle, vulgarisation, science, littérature.

Invité à prononcer un discours pour l'ouverture du nouvel amphithéâtre de la Faculté des sciences techniques nommé «Milutin Milanković», je me suis senti obligé de m'instruire un petit peu sur ce scientifique qui fait partie du trio mythique de la science serbe avec Nikola Tesla et Mihajlo Pupin. C'était le début d'une rencontre inattendue avec le livre intitulé À travers l'univers et les siècles. Une astronomie pour tous de 1928 et la découverte d'un transfert culturel inattendue là où les chercheurs en littérature ne s'aventurent que très rarement. Un transfert qui témoigne

*psekerus@ff.uns.ac.rs 
qu'écrire le monde en français ne veut pas dire obligatoirement en langue française mais qu'on peut l'écrire aussi par l'adoption des modèles et des manifestations culturelles françaises.

Les premières pages du livre mentionné paraissaient comme un déjà vu et assez rapidement il devient évident que le modèle pour le livre arrive du XVIIe siècle français et qu'il s'agit de l'œuvre de l'écrivain, scientifique et secrétaire de l'Académie des sciences, Bernard le Bovier de Fontenelle (16571757) et de ses Entretiens sur la pluralité des Mondes, dont la première édition paraît en 1686. Milutin Milanković a profité de ce modèle pour vulgariser des sujets d'astronomie dans le Royaume des Serbes, Croates et Slovènes entre deux guerres, de la même manière que Fontenelle le faisait au XVIIe siècle en France. Outre les thèmes de l'astronomie, Milanković prend également la forme de la prose épistolaire à une voix avec un seul correspondant, ou une correspondante plus exactement, avec la même argumentation qui explique ce choix, aussi bien que quelques réflexions sur le problème du travail de vulgarisateur. L'analyse comparative de ses deux ouvrages qui, utilisant des procédés semblables, explorent des zones frontalières entre la science et la fiction du XVII e et du XXe siècle s'imposait, et cela m'a poussé à faire cette petite recherche que je vais partager ici avec vous.

La littérature de vulgarisation d'abord. Daniel Raichvarg et Jean Jacques nous rappellent dans le livre Savants et ignorants que vulgus veut dire "peuple» et non "vulgaire», "que les langues 'vulgaires' sont les langues vivantes et que la Bible elle-même n'a pu se répandre dans le monde que grâce à la traduction qu'on nomme la Vulgate » (Raichvarg, Jacques, 1991 : 9).

D’après Le Dictionnaire Littré, c'est Mme de Staël qui, au début du XIXe siècle, a utilisé le mot "vulgarité », pour décrire des choses sans distinction. Mais rapidement, on réalise qu'un vulgarisateur ne fait que répandre la possession et l'usage d'un savoir-faire ou d'une connaissance sans distinction aux "gens du monde », aux classes moyennes, aux ouvriers, aux enfants ou aux femmes. Quoi qu'il en soit, le mot «vulgarisation » s'est imposé et son synonyme concurrent, «popularisation » est, lui, resté beaucoup moins... populaire (Raichvarg, Jacques, 1991 : 9).

Le livre de Fontenelle est le résultat d'une nouvelle situation au XVII ${ }^{\mathrm{e}}$ siècle où le nombre de publications scientifiques croît très rapidement et le niveau de ces publications s'élève aussi, alors que le public traditionnel n'arrive plus à suivre. "Les Traités de Newton, par exemple, ne se lisent plus comme un roman. [...] Ce fossé entre 'la science qu'élaborent certains' et 'la 
science que d'autres ne peuvent que recevoir' traverse toute l'histoire de la vulgarisation » (Raichvarg, Jacques, $1991: 8$ ).

Dans la Préface des Entretiens sur la pluralité des mondes Fontenelle essaye d'expliquer la recherche de ce double langage qui doit satisfaire les gens du monde, les lecteurs assidus de textes scientifiques, et les savants :

J'ai voulu traiter la philosophie d'une manière qui ne fût point philosophique ; j'ai tâché de l'amener à un point où elle ne fût ni trop sèche pour les gens du monde, ni trop badine pour les savants. Mais si on me dit, [...] qu'un pareil ouvrage n'est propre ni aux savants qui n'y peuvent rien apprendre, ni aux gens du monde qui n'auront point d'envie d'y rien apprendre [...] il se peut bien faire qu'en cherchant un milieu où la philosophie convînt à tout le monde, j'en aie trouvé un où elle ne convienne à personne ; les milieux sont trop difficiles à tenir, et je ne crois pas qu'il me prenne envie de me mettre une seconde fois dans la même peine [...] (Fontenelle, $1800: 3$ ).

Je dois avertir ceux qui liront ce livre, et qui ont quelques connaissances de la physique, que je n'ai point du tout prétendu les instruire, mais seulement les divertir, en leur présentant d'une manière un peu plus agréable et plus égayée ce qu'ils savent déjà plus solidement (Fontenelle, $1800: 5$ ).

Milanković de son coté, fait écho de ces paroles dans la première lettre de son livre: "Да, популарне астрономије треба да поучавају и да забављају. Но када уче, онда су досадне, а кад забављају, онда не уче, па зато испада, на концу крајева, обично ова комбинација : досадно и ненаучно » (Milanković, 1943 : 11).

[ « Oui, les astronomies populaires devraient enseigner et divertir. Mais quand elles enseignent, alors elles sont ennuyeuses, et quand elles divertissent, elles ne font pas apprendre, et après tout, d'habitude nous avons cette combinaison comme résultat : ennuyeux et non scientifique. »]

Ensuite il ajoute les phrases suivantes avec une certaine coquetterie qui semble dire ce que nous anticipions déjà : « У претсобљу мог кабинета на Универзитету стоји, покривен слојем прашине, један велики орман, препун таквих популарних астрономских дела; не бих желео да тог дебељка власторучно кљукам храном... $»^{1}$ (Milanković, 1943 : 11).

\footnotetext{
${ }^{1}$ Milanković a écrit de nombreux livres de vulgarisation scientifique et de l'histoire des sciences: Isak Njutn i Njutnova Principija (1946), Osnivači prirodnih nauka Pitagora Demokrit - Aristotel — Arhimed (1947), Istorija astronomske nauke od njenih početaka
} 
[ «Dans le vestibule de mon bureau à l'Université se dresse, recouvert d'une couche de poussière, un grand placard rempli de ces œuvres astronomiques populaires; Je ne voudrais pas gaver ce gros homme de ma propre nourriture... »].

Quelle est donc la solution que Fontenelle trouve entre « divertir » et «apprendre» et comment évite-t-il d'être ennuyeux tout en n'étant pas suffisamment scientifique ? Il présente les découvertes astronomiques dans un dialogue galant: un Philosophe, influencé par Copernic et par Descartes, entretient une jeune Marquise savante, et lui découvre, pendant six promenades nocturnes, les mouvements des astres et la pluralité des mondes habités, "mondes possibles » précise-t-il. Le charme de l'ouvrage tient à un mélange habile de considérations scientifiques et métaphysiques et de galanteries. Fontenelle l'organise comme roman épistolaire à une voix, composé de six soirées, c'est-à-dire, six lettres, comptes rendus à Monsieur L :

Vous voulez, Monsieur, que je vous rende un compte exact de la manière dont j'ai passé mon temps à la campagne, chez Madame la Marquise de $G^{* * *}$. Savez-vous bien que ce compte exact sera un livre; et ce qu'il y a de pis, un livre de philosophie ? [...] Je vous les [les entretiens] diviserai par soirs, parce qu'effectivement nous n'eûmes de ces entretiens que les soirs (Fontenelle, $1800: 8,10$ ).

Chaque lettre porte un titre qui annonce le thème discuté lors d'une soirée: Que la Terre est une planète qui tourne sur elle-même, et autour du Soleil, pour la première, Que la Lune est une Terre habitée pour la deuxième et ainsi de suite jusqu'à la sixième soirée avec la lettre intitulée Nouvelles pensées qui confirment celles des Entretiens précédents. Dernières découvertes qui ont été faites dans le Ciel. Pourquoi le choix de la forme épistolaire? Fontenelle l'utilisait déjà dans un roman intitulé Lettres galantes de chevalier d'Her**, de 1685. Une autre explication c'est que la forme épistolaire satisfaisait un besoin de fragmentation de la matière, permettant le traitement des sujets très vaguement reliés. Malheureusement, le dialogue que ce genre favorise n'est pas instauré entre les lettres, (il s'agit de la forme épistolaire à une voix), mais à l'intérieur des lettres qui sont des rapports, comme je l'ai déjà mentionné, ce

do 1727. (1948), Kroz carstvo nauke — slike iz života velikih naučnika (1950), 22 veka hemije (1953) i Tehnika u toku davnih vekova (1955). 
qui leur donne peu de vraisemblance. Une autre explication est que la popularité de la forme épistolaire, déjà utilisée par l'auteur, favorisait son emploi ici.

Et Milutin Milanković ? Il suit le modèle de son lointain prédécesseur. Son livre est né des lettres (une trentaine) des lettres publiées au cours de trois ans dans la revue Letopis Matice srpske dans lesquelles il dialogue avec une dame sur des thèmes allant de l'histoire de l'astronomie jusqu'aux voyages spatiaux. Ces lettres, avec six nouvelles ajoutées, sont mises en forme du livre en 1928, intitulé À travers l'univers et les siècles. Lettres d'un astronome.

Le livre de Milanković devient ainsi un roman épistolaire avec les éléments de récit de voyage imaginaire et réel, des mémoires intimes et même du roman de science-fiction.

Ses lettres remplacent une conversation sur l'histoire de l'astronomie mais l'auteur profite de cette occasion pour se remémorer de sa vie à Dalj, près d'Osijek, de sa scolarité à Osijek et à Vienne, de ses premiers succès et échecs dans le domaine professionnel, de son doctorat à Vienne et de ses voyages en Allemagne et en Hongrie, du travail à l'Université de Belgrade. Plusieurs lettres décrivent ainsi sa mission à Constantinople en 1923 où il devait proposer aux Congrès des églises orthodoxes la réforme du calendrier julien qui, pour des raisons politico-religieuses, n'a jamais été appliquée. Dans ces lettres le roman ressemble le plus aux mémoires.

Lorsqu'il traite de la science d'astronomie, l'écrivain utilise une approche très personnelle : son astronome part en voyage imaginaire avec une amie, à travers le temps et l'espace, aux endroits et les époques qui seront cruciaux pour le développement de l'astronomie.

Није ли боље да Вас, место да Вам причам и тумачим, узмем под руку и поведем на замишљена, али душевно доживела путовања у тајанствености васионе и векова? [...] За свако такво путовање можете се, по Вашем укусу, оденути и окитити. [...] Наш пут ће нас водити кроз васиону и векове, али и живот земаљски. Ето, такву, без утврђеног плана и система, више поверљиву него научну, замишљам нашу преписку. Да ли би Вам се она допала (Milanković, 1943 : 12)

[Au lieu de parler et d'interpréter, n'est-ce pas mieux, de vous prendre par la main et de vous emmener dans des voyages imaginaires, mais vécus avec émotions, dans le mystère de l'univers et des siècles? [...] Pour chacun de ces voyages, vous pouvez, selon votre goût, vous habiller et vous embellir. [...] Notre chemin nous conduira à travers l'univers et les siècles, mais aussi à travers la vie terrestre. C'est de cette façon, sans plan ni système établi, plus 
confidentiel que scientifique, que j'imagine notre correspondance. Aimeriezvous cela?]

Cette prise de main de la dame est fictive, épistolaire, aussi bien que leur voyage à travers le temps. Vêtus de vêtements de l'époque choisie, ils se promènent à Babylone, berceau de l'astronomie, dans l'Athènes antique, en Alexandrie, Dubrovnik, Padoue, Prague, en Angleterre, ou encore en Allemagne. Cachés aux yeux des indigènes, ils assistent, comme dans une salle de théâtre, aux dialogues des prêtres babyloniens, d'Aristote, Eratosthène et autres grands scientifiques d'Alexandrie, de Cléopâtre et de César, de Galilée et de Newton, ces dialogues qui changeront pour toujours la science de l'astronomie. Parfois, les lettres décrivent des expériences, suivent le chemin d'une écriture ancienne ou le développement d'un instrument scientifique, ou décrivent l'architecture des villes anciennes et nouvelles. L'histoire des idées scientifiques est entremêlée de connaissances de base sur notre soleil et nos planètes et leurs orbites. Dans la partie centrale du livre Milanković présente ses postulats sur le changement climatique, c'est-à-dire sur la nature cyclique des périodes glaciaires à travers l'histoire et l'avenir de notre planète Terre.

Enfin, dans les dernières lettres, le roman épistolaire prend l'allure d'un roman de science-fiction vernien. Notre astronaute invite son ami à faire avec lui un voyage extraterrestre dans un train fusée vers la Lune d'abord, puis vers Mars et enfin Venus. La 37ème et dernière lettre de la deuxième édition serbe, nous amène en un pèlerinage vers la Voie lactée, vers des amas d'étoiles et des essaims de soleils. Alors que les temps révolus et les voyages à travers l'espace sont décrits de manière experte, l'écrivain se révèle ici l'auteur de science-fiction, variant encore une fois le genre de ses contes en lettres.

Analysant la littérature de vulgarisation Joël Castonguay-Bélanger dit que :

[...] plusieurs fictions dialoguées mettent [ici] en scène une initiation intellectuelle entre au moins deux personnages: d'un côté, une figure de pédagogue en possession d'un savoir à transmettre; de l'autre, un destinataire ignorant ne demandant qu'à être éclairé. La relation pédagogique prend tantôt la forme d'une conversation galante ou familière, tantôt celle d'un échange épistolaire, et exploite à divers degrés la scénographie et le dispositif narratif mis en place. Parce qu'on les représente comme l'incarnation idéale d'un esprit vierge à instruire, les femmes et les enfants apparaissent souvent comme les destinataires privilégiés du discours pédagogique. [...] les exemples abondent où les interlocuteurs se 
voient réduits à leur seul prénom ou se contentent d'interactions minimales qui donnent à la structure dialogique le statut de prétexte narratif inexploité (Castonguay-Bélanger, 2014 : 177).

Fontenelle et Milanković ne s'écartent pas de cette pratique mais l'appliquent de manière cohérente. Le premier justifie de la façon suivante son choix d'une aimable marquise comme interlocutrice :

J'ai mis dans ces entretiens une femme que l'on instruit et qui n'a jamais ouï parler de ces choses-là. J'ai cru que cette fiction me servirait à rendre l'ouvrage plus susceptible d'agrément, et à encourager les dames par l'exemple d'une femme qui, ne sortant jamais des bornes d'une personne qui n'a nulle teinture des sciences, ne laisse pas d'entendre ce qu'on lui dit, et de ranger dans sa tête, sans confusion, les tourbillons et les mondes (Fontenelle, $1800: 31$ ).

Il ajoute aussi :

Il est vrai que les idées de ce livre-ci sont moins familières à la plupart des femmes que celles de la Princesse de Clèves, mais elles n'en sont pas plus obscures, et je suis sûr qu'à une seconde lecture tout au plus, il ne leur en sera rien échappé (Fontenelle, 1800 : 5).

L'habitude de parler de science à un public spécifiquement féminin traverse toute l'histoire de la vulgarisation depuis ses origines. Cependant, les femmes s'y affirment non seulement comme lectrices ou comme interlocutrices privilégiées de l'œuvre vulgarisatrice, mais aussi comme auteurs. Il suffit de mentionner Mme du Châtelet (Institution de Physique), traductions et commentaires de Newton en français ou Mme d'Épinay (Conversation d'Émilie), mais dans les interprétations de cette pratique le côté misogyne l'emporte le plus souvent comme, par exemple, chez Sainte-Beuve d'après qui ce détour par un disciple féminin était, pour ainsi dire, obligé car Fontenelle s'adressait alors à son meilleur public, c'est-à-dire « à des esprits plutôt vides et vacants que déjà occupés par d'opiniâtres erreurs. Mieux valait avoir affaire à un ignorant certes qu'à un esprit encroûté, entêté de la vieille science » (Sainte-Beuve, 1868 : 97). Presque trois siècles plus tard, Milanković continue cette tradition d'interlocutrice féminine et comme son prédécesseur lui tient des propos galants et fait la cour à son élève. Une certaine condescendance aimable, fontenellienne dirais-je, ne lui est pas étrangère non plus, comme dans les phrases suivantes : 
Дама: „Но она (астрологија, прим. аут) је, јел'те, у последње време дошла опет у моду, и многи верују у њу.“ - „Мода! Законик жене! Истина је, астрологија стоји у високој конјунктури, свет је преплављен проспектима астролога. Будуһност је постала опет траженом робом, иначе ови силни астролози не би имали од чега да живе" (Milanković, $1943: 31)$.

La dame: [«Mais elle (l'astrologie, N. d. A) est revenue à la mode ces derniers temps, et beaucoup croient en elle.»- «Ah la mode! Loi pour les femmes! C'est vrai, l'astrologie est très sollicitée, le monde est inondé de perspectives d'astrologues. L'avenir est redevenu une marchandise recherchée, sinon ces puissants astrologues n'auraient rien pour vivre. » ]

De nombreux procédés empruntés à Fontenelle par Milanković surprennent beaucoup moins que l'étonnante similitude de destins de deux auteurs. Non seulement que leurs vies se ressemblaient, (les deux étaient membres influents des Académies des sciences de leurs pays, l'un comme secrétaire à vie et l'autre comme vice-président), mais ils sont restés liés même après la mort. ${ }^{2}$ L'Union Astronomique Internationale a donné en 1970 le nom de Milanković à un cratère de la Lune et en 1973 à un astéroïde nommé 1605 Milanković. Fontenelle a reçu ces honneurs un peu plus tôt, en 1935 quand un cratère de la Lune a reçu son nom, aussi bien qu'un astéroïde nommé 10069 Fontenelle.

Pour conclure, nous pouvons dire que, malgré ses hésitations, Milanković a décidé de nourrir avec « sa propre nourriture » son grand placard poussiéreux rempli des œuvres astronomiques populaires, qui se trouvait dans le vestibule de son bureau universitaire. Il sortira de ce placard le livre de Fontenelle Entretiens sur la pluralité des mondes de 1686 et l'utilisera comme modèle pour son livre de vulgarisation scientifique $\grave{A}$ travers l'univers et les siècles de 1928. Tous les procédés déjà employés par Fontenelle se retrouvent

${ }^{2}$ En l'honneur de ses réalisations en astronomie, un cratère d'impact de l'autre côté de la Lune a reçu le nom de Milanković lors de la 14e Assemblée générale de l'Union Astronomique Internationale en 1970. Son nom est également donné à un cratère sur Mars lors de la 15e Assemblée générale de l'UIA en 1973. Depuis 1993, la médaille Milutin Milanković a été décernée par la Société européenne de géophysique (appelée EGU depuis 2003) pour ses contributions dans le domaine du climat à long terme et de la modélisation. Un astéroïde de la ceinture principale découvert en 1936 a également été surnommé 1605 Milankovitch. En 1935 le nom Fontenelle est donné à un cratère lunaire et le nom (10069) Fontenelle, à un astéroïde. (Milutin Milanković (Милутин Миланковић), (n.d.)). 
dans ce livre : le genre de roman épistolaire à une voix, mais plus complexe et plus exigeant que celui de son prédécesseur. Son interlocutrice sera une femme charmante, selon les règles du genre de vulgarisation et de son modèle. Le maître Milanković va profiter de sa position, aussi bien que le maître Fontenelle, et va faire discrètement la cour à son élève. Cela faisait partie de la stratégie annoncée par Fontenelle et adoptée par son successeur : instruire et divertir en même temps, et surtout ne pas ennuyer. Apparemment, vu la réception des ouvrages, les deux ont réussi dans leurs entreprises : Le livre de Fontenelle a eu plus d'une trentaine d'éditions jusqu'à aujourd'hui et de nombreuses traductions; celui de Milanković a, pour sa part, eu une dizaine d'éditions en Serbie et deux en Allemagne, pendant sa relativement courte vie de 90 ans.

Pavle Sekeruš, Ivana Živančević Sekeruš

\section{MILUTIN MILANKOVIĆ AND HIS FRENCH READINGS}

\section{Summary}

The works of Milutin Milanković, mathematician, astronomer and climatologist, which, for the uninitiated, carry enigmatic titles and speak of thermal phenomena and solar radiation, would have had little chance to attract the attention of literary critics, if among them one had not found some popular science works intended for the general public. One of them, titled Kroz vasionu i vekove (Across the Universe and Centuries) from 1928, a popular epistolary novel, reveals the author's French readings fairly quickly. The influence of the 17th century French author Bernard le Bovier de Fontenelle and his book Interviews on the Plurality of Worlds from 1686 seems obvious, however neither scientists nor literary historians say much about it. The subject of this paper is comparative analysis of these two books, which use similar writing strategies, exploring border areas between 17th and 20th century science and fiction. Our objectives are to place the mentioned works in the scientific and cultural context of the respective periods. Furthermore we want to take a new look at literary texts with a strong scientific content and analyze the phenomenon of popularization of science, as well as highlight often unknown relationships between literature and science.

Key words: Milanković, Fontenelle, popularization, science, literature. 


\section{RÉFÉRENCES BIBLIOGRAPHIQUES}

Castonguay-Bélanger, J. (2014). À l’ombre de Fontenelle. Dissémination du discours scientifique par la fiction au XVIIIe siècle. Littératures classiques, 2014/3, 85. 171-187.

Fontenel, B. l'B. de. (1997). O mnoštvu svetova. Beograd : Paideia.

Fontenelle, B. l'B. de. (1800). Entretien sur la pluralité des mondes. Lyon : Leroy. Littré, É. (1863-1877). Dictionnaire de la langue française. Paris : Hachette. Consulté le 15 octobre 2020, disponible sur http://littre.reverso.net/ dictionnaire-francais/

Milanković, M. (1943). Kroz vasionu i vekove. Jedna astronomija za svakog. Beograd : Jugoistok. (Миланковић, М. (1943). Кроз васиону и векове. Једна астрономија за сваког. Београд : Југоисток.)

Raichvarg, D.-Jacques, J. (1991). Savants et Ignorants. Une histoire de la vulgarisation des sciences. Paris : Seuil.

Sainte-Beuve. (1868). Nouveaux lundis. Tome 10. Paris : Michel Lévy frères. Consulté le 10 octobre 2020, disponible sur https://gallica.bnf.fr/ ark:/12148/bpt6k6121137p.texteImage

Milutin Milanković (Милутин Миланковић). (n.d.). In : Wikipedia. Consulté le 10 septembre 2010, sur https://sr.wikipedia.org/wiki/Милутин_ Миланковић 